\title{
Evaluation of Body Area Networks
}

\author{
Sajad Ahmadian Naybin ${ }^{1} \&$ Yosef Farhang ${ }^{2}$
}

${ }^{1}$ Faculty of Engineering, Department of Computer, Software Engineering, Islamic Azad University of Urmia, West Azerbaijan, Iran

${ }^{2}$ Faculty of Engineering, Department of Computer, Software Engineering, Islamic Azad University of Urmia, West Azerbaijan, Iran

Correspondence: Sajad Ahmadian Naybin, Faculty of Engineering, Department of Computer, Software Engineering, Islamic Azad University of Urmia, West Azerbaijan, Iran.

Received: October 30, 2017

Accepted: November 16, 2017

Online Published: November 30, 2017

doi:10.5539/res.v9n4p177

URL: http://doi.org/10.5539/res.v9n4p177

\begin{abstract}
Body Area Network, due to recent advances in wearable sensors, intelligent surveillance with small size and low weight are made in low power consumption. Human physiological activities are constantly monitored by sensors. In this article, key controversies surrounding the field of BAN communications, including layers, and for example, the patient's health status and patterns of movement and etc.
\end{abstract}

Keywords: (Body Area Network) ‘(Intra-BAN communication) ‘(Inter-BAN communication) ‘(Beyond-BAN communication).

\section{Introduction}

BAN- Body Area Network is composed of three layers, which include Intra-BAN communication, Inter-BAN communication, and Beyond-BAN communication. Body sensor with advances in circuit design, signal have progressed. MAC layer parameters are named as well as technologies and projects. Overall, this network is used for the patient's health status and patterns of movement and sports, and so on.

\section{Communication of Architecture BAN}

Wireless networking technology that available on WLANs are differences with BANs as follows:

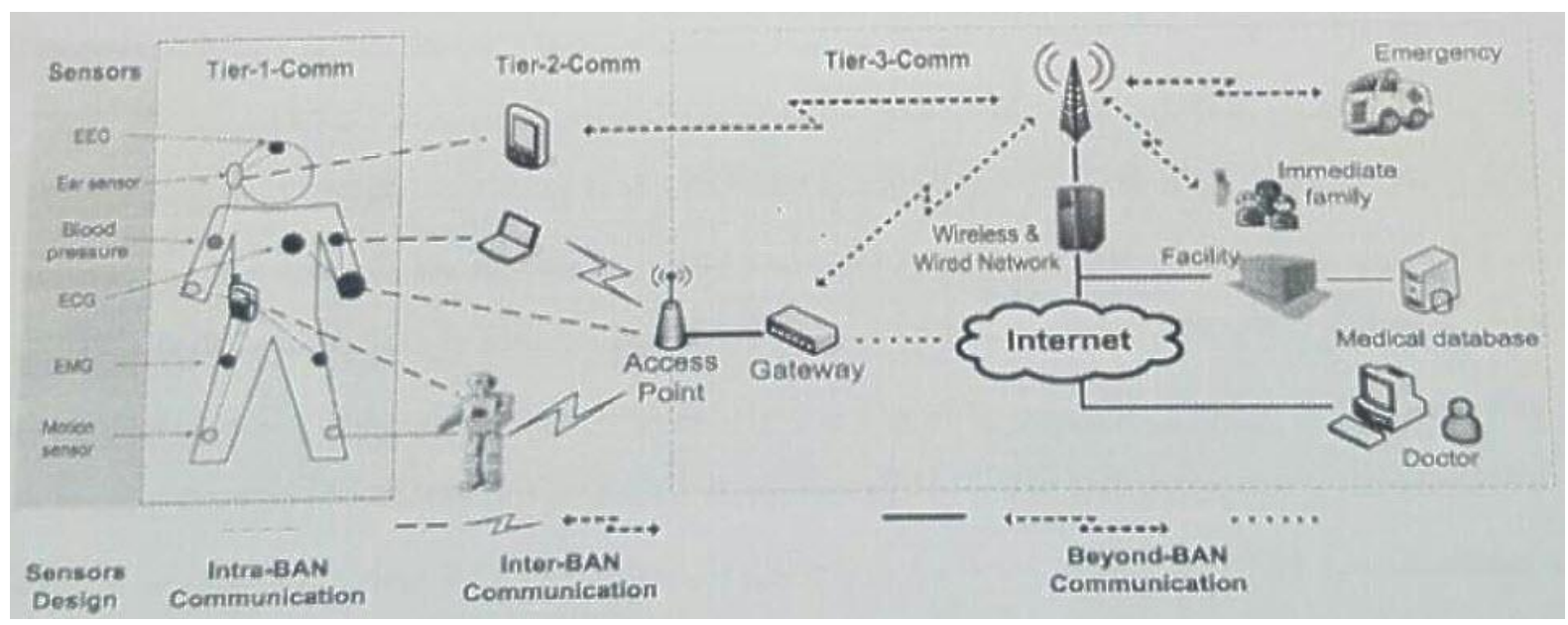

Figure 1. describes a general architecture of a health monitoring system is based on the BAN.

ECG, EEG, EMG, motion sensors and blood pressure sensors that their data on a PS-personal server sends adjacent. Then, via Bluetooth or WLANs, the data is sent to faraway places of a physician to determine the immediate in a real-time. History in the database for storing medical or similar equipment to send emergency alerts. In this article, BAN communications architecture is divided into three components: 
First layer: Intra-BAN communication

Second layer: Inter-BAN communication

Third layer: Beyond-BAN communication.

Figure 1. BAN communication system with three-layered architecture

Figure 1. Covering the range of several components that are described that at low levels and high levels indicate and facilitating the creation because of these components, BAN efficient systems for a wide range of applications. Each component is designed to formalize.

\subsection{First layer: Intra-BAN Communication}

For the introduction of the Intra-BAN communication in reference to radio communications, about two meters around the human body, which is also divided into the following categories. As seen in figure 1, (1) there are communications between sensors and body, (2) there are communications between personal devices, body sensors, and (PS) Portable (eg Mobile PDA, etc) that have a direct communication.

Body sensors and network BAN, design communication within the Intra-BAN communication, which are very sensitive. Furthermore, battery failure itself, and features low bit rate sensor in the device body issues are challenging for designing a MAC protocol capable of efficiently providing a QoS- quality of service. In this issue, we avoid the challenges of connecting wireless sensors and personal devices plans which are available.

As MITHril (the books encompass health - wearable medical technology page 42 to 49) (Pentland A, 2004) and Smart (The development of networking body monitor the physiological signals in the emergency room of waiting area) (Curtis D. Shih, Waterman J. Guttag, Bailey. Etal, 2008) that cables used in directly linking smart. Various commercially available sensors with a personal device (PS), such as a mobile phone or PDA is shown in Fig a2.

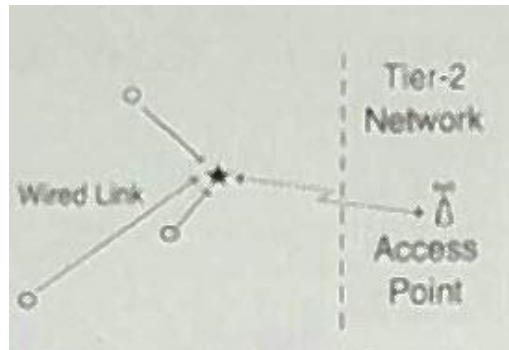

(a)

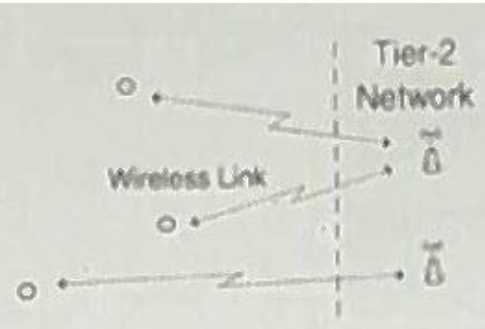

(b)

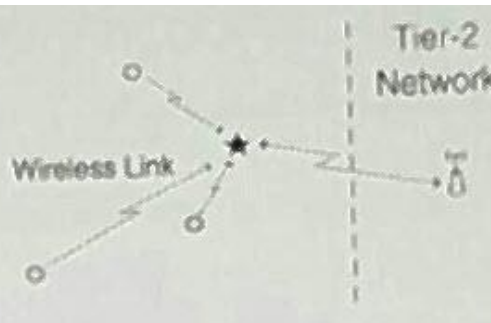

(c)

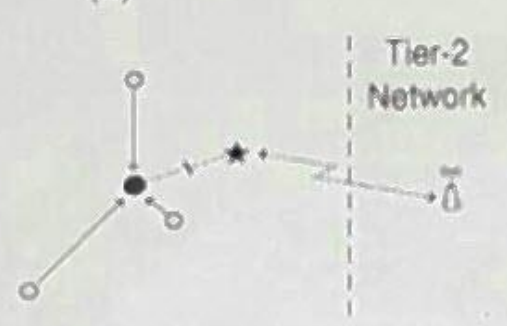

(d)

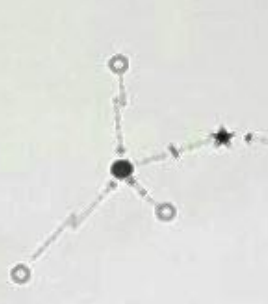

Personal server

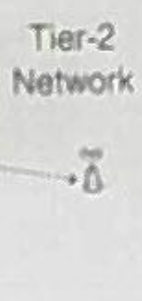

(e)

Figure 2. communication between PS and PDA

Alternatively, or in turn, guidelines or blue codes (technical report TR-08-05 of Harvard University in the field of sensor networks for healthcare) (Shnayder.V.Chen, Lorincz.K, Fulford-jones, Welsh. M, 2005) indicating, as shown in figure b2 direct connection of sensors and Apps-access points without a personal device (PS). Compare the two previous comment form is $\mathrm{c} 2$. Providing varied architecture in order to use a star topology (STAR), by the various sensors signal the body to take a PS for the physiological data obtained their transition to an access point (Barth A, Wilson S, Hanson M, Powell H,Unluer D, Lach J, 2008). D2 and e2 Fig is progressing a BAN on two levels. In the first level, wireless communication or wired sensors in a variety of processor instructions inside center in the delivery of raw data and the ability to hold (save). After synthesizing the data, in terms of the necessity of the data, transmitted by the CPU (central processor) and delivered. The personal devices (PS) have many challenges to the solutions, as we enter the advanced sensor data processing, with a view to properties for 
biomedical communications. For example, Baker CR, Armijo K, Belka S, Benhabib M, Bhargava V et al (2007) accepted everywhere Intra-BAN base two-level structure of a node receives. Message away by sensors to other complexes, which is connected to the base station, as you can see in fig 2. It is obvious that fig architect processing e2 has the complexity of Fig system increases to a2.

\subsection{Second Layer: Inter-BAN Communication}

WSNs cannot be separated and they are commonly worked as the rotating system, BAN rarely works alone. The price we establish a connection between PS and define one or more AP. Access profound spread on the part of the infrastructure or in a strategic location in dynamic environments are address to the emergency. Similarly, the network layer, as seen in figure 1. BAN connected to each other by various networks that are used daily access easily. Such as the Internet and other networks.

We have divided pattern of Inter-BAN communication in two categories, the basic architecture Infrastructure (Figure 3) and the architecture of the base or ad hoc based architecture (Figure 4) when the basic architecture infrastructure was provided. The wide bandwidth with flexibility and centralized control there, since that was spreading quickly, the architectural base has been easy, and we face a dynamic environment. Such as responding to emergency medical care or go to the accident site (Baker CR, Armijo K, Belka S, Benhabib M, Bhargava V et al, 2007).

\subsubsection{Infrastructure Based Architecture}

More BAN applies the communication infrastructure between the body areas networks (Inter-BAN communication) to the surrounding environment or it present in our environment. With limited extent, for example in a hospital waiting room, home office, toward or against something similar networks, basic network infrastructure advantages offered by centralized management and control of security. Because of this central structure, AP also works as a database service that includes different applications (Curtis D, Shih, E, Waterman J,Guttag, Bailey J et al 2008), (Jiang S, Cao Y, Lyenger S, Kuryloski P, Jafari R,Xue Y,Bajcsy R, Wicker S 2008)

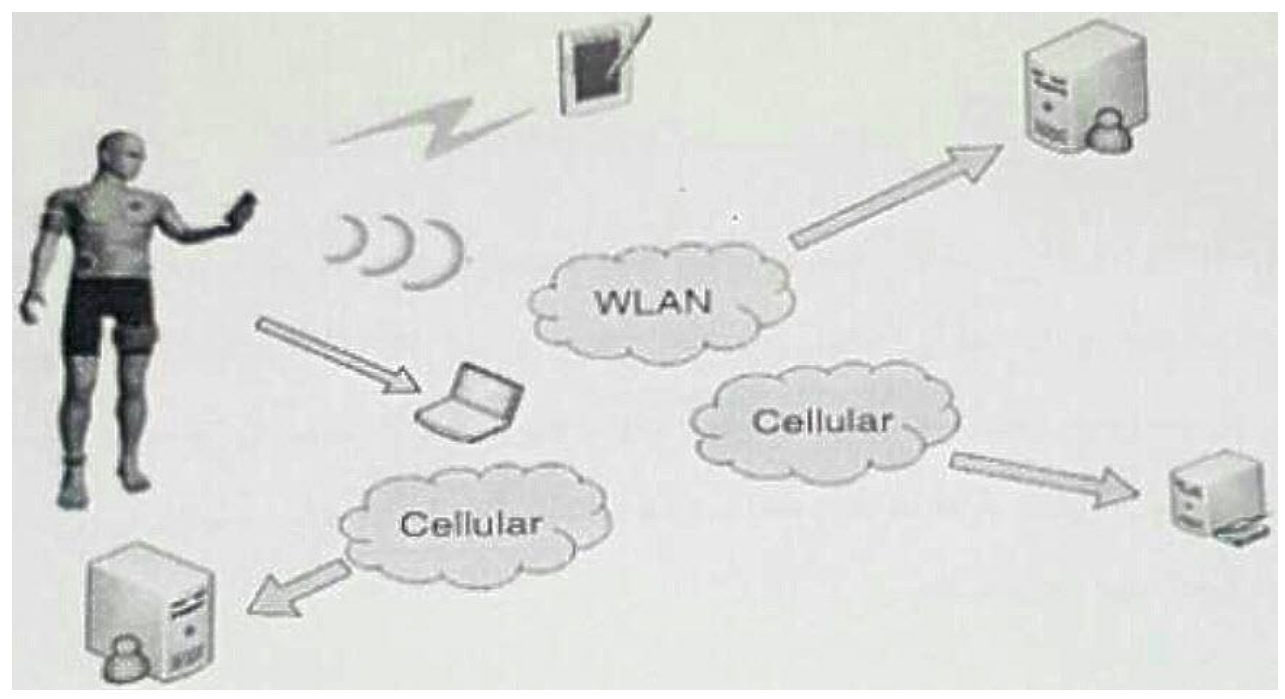

Figure 3. Infrastructure based architecture

\subsubsection{Ad hoc Based Architecture}

At the base architecture, AP various body sensors have been developed to assist in the transfer of information within the medical center. Thus, the service area under great visibility, relative to the basic architecture of the infrastructure. Users can easily move around the building, playground or be rescued immediately in an emergency event. If the BAN network coverage of about two meters. Through the development of connections between (for the same AP) almost one hundred meters. Installation for both short-term and long-term installation (such as houses) are suitable. 


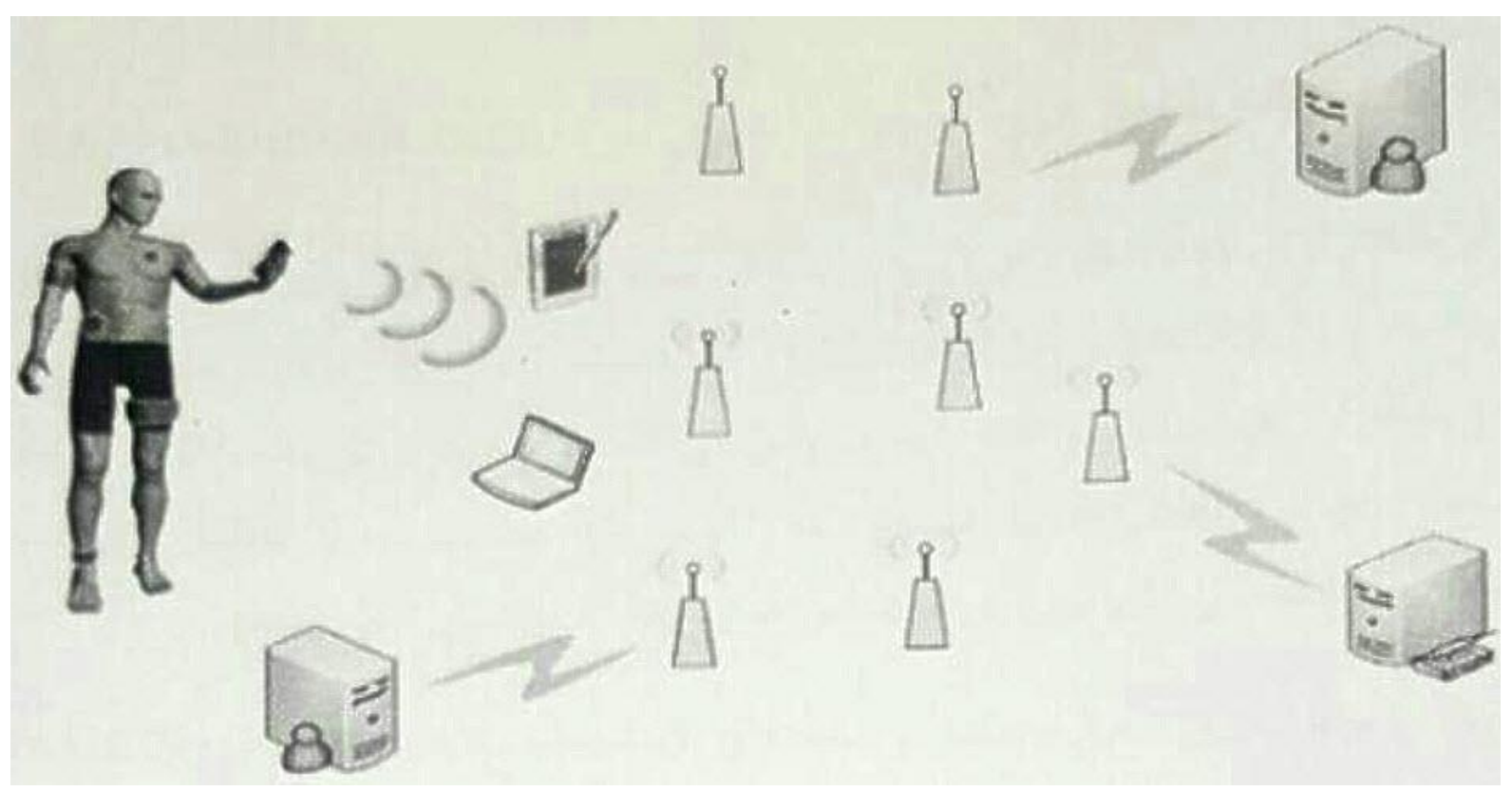

Figure 4. Ad hoc based architecture

There are two categories of nodes. The installation site or actuator nodes and sensor/actuator in the human body and the routing nodes, which are around a BAN.

They both have the hardware to facilitate of the routing multi-stage radio (micro radio frequency). Install the same architecture for wireless sensor networks (WSN) is traditional, and both of them have been used repeatedly as an interface to the outside world in WSN, which all sensor nodes or node are routers yet. Hence, they are a single radio message; all communications have the same bandwidth. A concussion occurs so easily. To determine the number of nodes in sensor/actuator and navigation in certain areas usually use a mechanism asynchronous MAC. It is known as CSMA/CA- carrier sense multiple access with collision avoidance, in ZigBee/IEEE 802.15.4 and dealing with collisions. AP variety of these systems has a mesh structure or network. The features are:

(1) An extensive radio coverage is achieved, so that a multi-phase release of the data, allowing the best backup patients planets.

(2) Wireless high-speed, flexible can be accessed with a quick emergency response systems, for example, different routing nodes that can spread to the wall (Baker CR, Armijo K, Belka S, Benhabib M, Bhargava V et al 2007) or along the emergency routes (6) and (3) can easily spread without the effect of the integrated networks and the AP may whenever necessary to be added. Inter- BAN communication technology includes WLAN, Bluetooth, Zigbee, cellular, 3G Zigbee protocol's ability to support mesh networks. AP WLAN networks with higher speed than cellular networks or groups of the dwelling.

\subsection{Third Layer: Beyond-BAN Communication}

Compare the second and third layer is to figure out how to use communication design in urban areas. In the Connection Settings for these two networks (inter-BAN and beyond-BAN), the gateway is required (Gatewaydissimilar network connection between two computers, two different distinctly communication network to connect). The third layer can facilitate communication coverage rates and schedules an E-health system. The next step is equipped with a licensed healthcare professionals (eg, doctor or nurse) and doesn't have access to patients' medical information via the Internet and cellular networks. Layer beyond-BAN is an important component of the database. The personnel files and medical history database to support the maintenance and preservation. According to doctor's access and priority customer service doctor to inform users that need access. Each time, based on the data relative to the patient, automatic alerts can be sent via remote communication of different abilities. 


\section{The Body Sensors}

With the advances in circuit design, signal, MEMS-Micro-Electro Mechanical System and body sensors were smaller and wearable devices.

Some of the commercially available sensor devices in the area of the body are such as following:

Accelerometer 'blood glucose ‘blood pressure ‘carbon dioxide CO2 ، ECG ‘EEG ‘EMG 'gyroscope ‘pulse oximetry, in addition, many types of sensors that are used in network WSN.

Table 1. Sensors commonly employed BAN systems and their typical data rates

\begin{tabular}{lll}
\hline Sensor & Topology & Data rate \\
\hline Accelerometer/gyroscope & Star & High \\
Blood glucose & Star & High \\
Blood pressure & Star & Low \\
CO2 gas sensor & Star & Very low \\
ECG sensor & Star & High \\
EEG sensor & Star & High \\
EMG sensor & Star & Very High \\
Pulse oximetry & Star & Low \\
Humidity & Star & Very Low \\
Temperature & Star & Very Low \\
Image/video sensor & P2P & Very High \\
\hline
\end{tabular}

\section{Sensor Node}

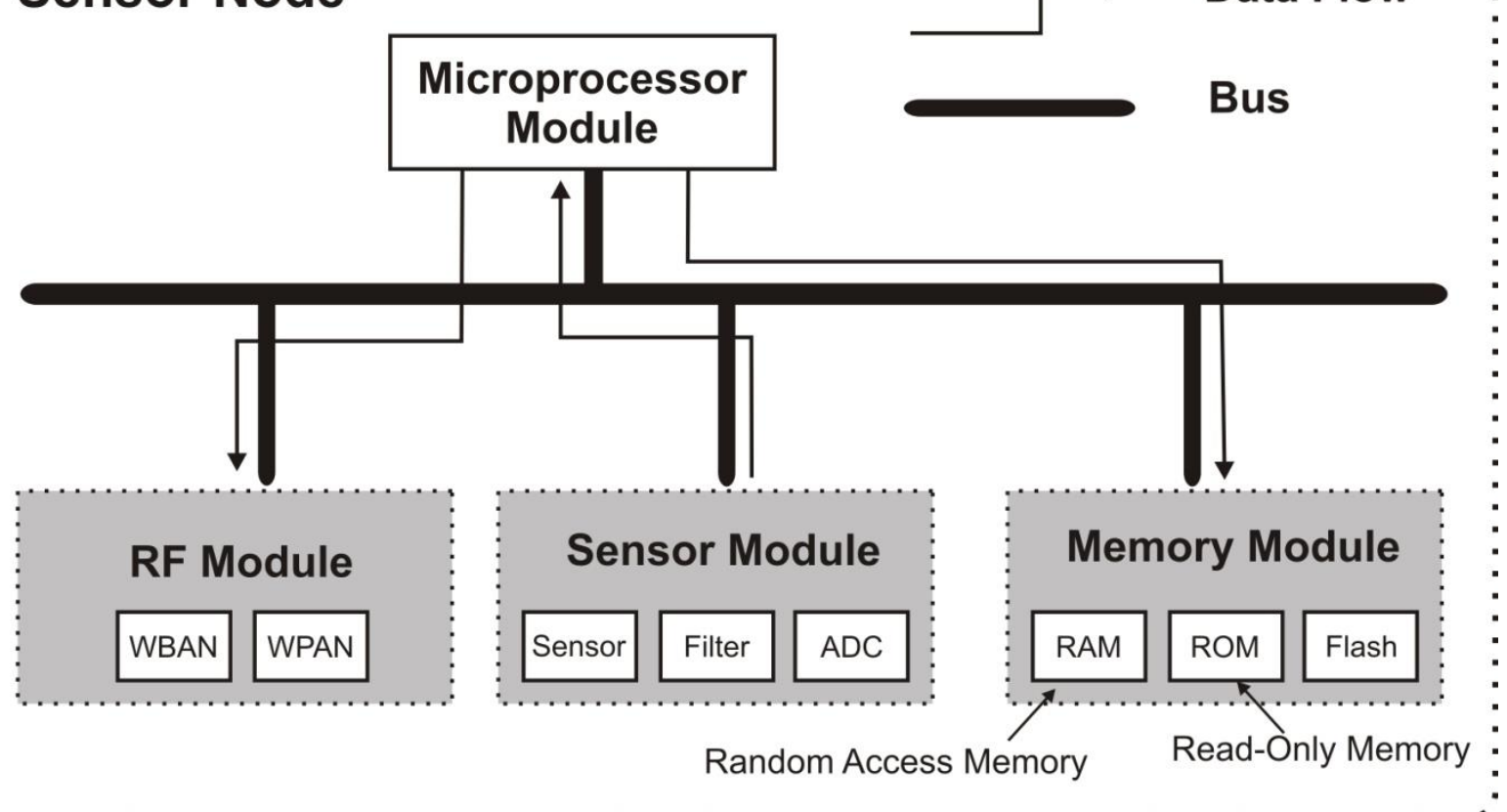

Figure 5. Sensor node 


\begin{tabular}{lllllll}
\hline Name & OS support & Wireless standard & $\begin{array}{l}\text { Data } \\
(\mathrm{kbps})\end{array}$ & $\begin{array}{l}\text { rate } \\
(\mathrm{m})\end{array}$ & $\begin{array}{l}\text { Outdoor } \\
\text { range }\end{array}$ & $\begin{array}{l}\text { Power } \\
\text { level }\end{array}$ \\
\hline BAN node & TinyOS & IEEE 802.15.4 & 250 & 50 & Low \\
BTNode & TinyOS & Bluetooth & - & - & Low \\
eyesIFX & TinyOS & TDA5250 & 64 & - & Low \\
iMote & TinyOS & Bluetooth & 720 & 30 & Low \\
iMote2 & TinyOS or & IEEE 802.15.4 & 250 & 30 & Low \\
& .NET & & & & Low \\
IRIS & TinyOS & IEEE 802.15.4 & 250 & 300 & Low \\
Mica2 & TinyOS & IEEE 802.15.4 & 250 & $75-100$ & Low \\
Mica2 & TinyOS & IEEE 802.15.4 & 384 & $>100$ & Low \\
Mulle & TCP/IP or & Bluetooth or & - & $>10$ & Low \\
TelOS & TinyOS & IEEE 802.15.4 & & & Low \\
ZigBit & TinyOS & IEEE 802.15.4 & 250 & $75-100$ & 3,700 & \\
\hline
\end{tabular}

\section{Physical Layer}

modeling of channels are written in refrences 9, 10,11 and 12. Antenna design for sensors in the body that divided into two groups.

1. Electric antenna, such as: dipole

2. Magnetic antenna, such as: loop

Antennas that are placed inside the human body should only make of non-perishable materials and biocompatibility with the body, such as; platinum or titanium circular of spiral fig antenna.

\section{MAC layer}

MAC layer here offs between reliability, downtime or latency and energy consumption in terms of the necessity to be able to decide.

Obviously: (Quality of Service- QoS), reliability, downtime or latency works on the executive career, which is reflected in energy consumption, complexity, and a mix of protocols.

MAC protocols efficient energy sources as following: 32, 31, 60, 24, 39, 13, 40, 61

Providing QoS for layer MAC: Some BAN networks require instant communication (real-time) as following: That's why we use ECG sensors.

MAC protocol for data transmission with QoS has three components:

1. control entry or admission

2. Scheduler QoS

3. Virtual MAC.

Distributed Queuing Body Area Network- DQBAN, general of source 8 in purpose MAC protocol which is the network supports QoS.

- Working with the IEEE 802.15.4 standard.

- The timing and control algorithms allow time.

- CAPs- contention access periods used for GTSs- guaranteed time slots and CFPs- contentionfree periods.

- Working with the IEEE 802.15.6 standard.

- Working with the IEEE 802.15.4 standard and combine, those with the new radio technology applied, 
such as WB-Ultra-Wideband.

\section{The Radio Technology}

- Bluetooth

- Bluetooth low energy technology

- ZigBee and IEEE 802.15.4 standard

- UWB and IEEE 802.15.6 standard

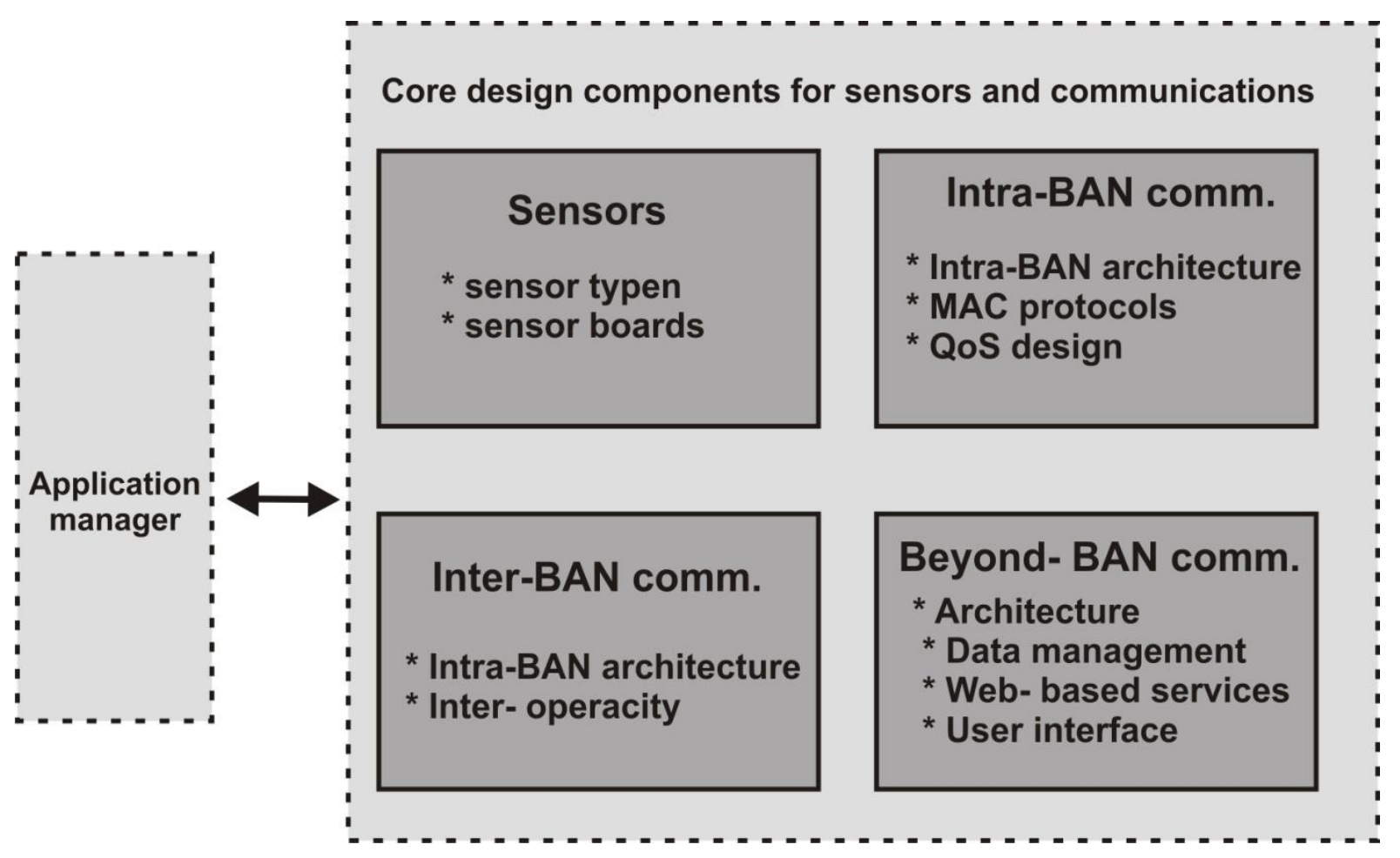

Fig6. Communications of sensor components

\section{Completed Projects}

1. CodeBlue

2. AID-N

3. SMART

4. CareNet

5. ALARM-NET

6. BAN (based intelligent system) and intelligent biosensor system for vehicle area networks 


\begin{tabular}{|c|c|c|c|c|c|}
\hline Projects & Sensors & $\begin{array}{c}\text { Intra×BSN } \\
\text { communication }\end{array}$ & $\begin{array}{c}\text { Inter-BSN } \\
\text { communication }\end{array}$ & $\begin{array}{l}\text { Beyond-BSN } \\
\text { communication }\end{array}$ & $\begin{array}{l}\text { Targeted } \\
\text { Application }\end{array}$ \\
\hline CodeBlue & $\begin{array}{l}\text { Puise oximeter } \\
\text { EKG motion }\end{array}$ & Wired & Mesh \& Zlgbee & N/A & Medical caro \\
\hline AID-N & $\begin{array}{l}\text { Pulse, Blood, } \\
\text { Temperaturo,ECG }\end{array}$ & Wired & Mesh \& Zigbee & $\begin{array}{l}\text { IntemelWiF|t } \\
\text { Cellular Networks }\end{array}$ & $\begin{array}{l}\text { Mass casualty } \\
\text { incident }\end{array}$ \\
\hline SHART & $\begin{array}{c}\text { ECG } \\
\text { SpO2 sensor }\end{array}$ & Wired & 802.110 & N/A & $\begin{array}{l}\text { Heatth monitoring } \\
\text { In waiting room }\end{array}$ \\
\hline CareNet & $\begin{array}{c}\text { Tritaxial } \\
\text { accelerometerl } \\
\text { gyroscope }\end{array}$ & $N / A$ & Zigbee & $\begin{array}{l}\text { Multihop } 802.11 / \\
\text { Internet }\end{array}$ & $\begin{array}{c}\text { Remote } \\
\text { healthcare }\end{array}$ \\
\hline ASNET & $\begin{array}{l}\text { Blood pressure, } \\
\text { temperature }\end{array}$ & Star topology & GPRS/GSM & $N / A$ & $\begin{array}{l}\text { Remote health } \\
\text { monitoring }\end{array}$ \\
\hline MrTHril & ECG, EKG & Wired & WiFi & $N / A$ & Healthcare \\
\hline WHAS & $\begin{array}{l}\text { ECG, EMG, } \\
\text { EEG, SpO2 \& } \\
\text { motion sensor }\end{array}$ & Star topology & $\begin{array}{l}\text { WLAN/Blueloo!h/ } \\
\text { GPRS }\end{array}$ & Intemet & Telemedicine \\
\hline WHOCA & $\begin{array}{c}\text { Tri-axial } \\
\text { acceleromater }\end{array}$ & $\begin{array}{l}\text { Star topology \& } \\
\text { Tinie fable-based } \\
\text { MAC protocol }\end{array}$ & Bitutooth & $\begin{array}{c}\text { Internet' } \\
\text { BlueloothWiFit } \\
\text { Ce'lular Networks }\end{array}$ & $\begin{array}{c}\text { Gesture } \\
\text { detection/sport }\end{array}$ \\
\hline MIMOSA & $\begin{array}{l}\text { Any sensors/ } \\
\text { RFID sensor }\end{array}$ & $\begin{array}{c}\text { WibreeiBluelooth } \\
\text { RFID }\end{array}$ & Cellufar Networks & Internet & $\begin{array}{c}\text { Ambient } \\
\text { intelligence }\end{array}$ \\
\hline
\end{tabular}

\section{Acknowledgments}

Fig 7. Previous projects

Thanks to Professor Masdari

\section{References}

Pentland, A. (2004). Healthwear: medical technology becomes wearable. Computer, 37(5), 42-49. https://doi.org/10.1109/MC.2004.1297238

Curtis, D., Shih, E., Waterman, J.,Guttag, Bailey, J. et al (2008). Physiological signa monitoring in the waiting areas of an emergency room. In: Proceedings of BodyNets 2008. Tempe, Arizona, USA.

Shnaydar, V., Chen, B., Lorincz, K., Fulford-Jones, T. R. F., \& Welsh, M. (2005). Sensor networks for edical care. Harvard University Technical Report TR-08-05.

Barth, A., Wilson, S., Hanson, M., Powell, H.,Unluer, D., \& Lach, J. (2008). Body-coupled communication or body sensor networks. The 3rd international conference on body area networks (BodyNets) empe, Arizona. https://doi.org/10.4108/ICST.BODYNETS2008.2964

Baker, C. R., Armijo, K., Belka, S., Benhabib, M., Bhargava, V. et al (2007) Wireless sensor networks for home healthcare. In: International conference on advanced information networking and applications workshops, AINAW'07, 832-937. https://doi.org/10.1109/AINAW.2007.376

Body Sensor Networks. Available at: http://ubimon.doc.ic.ac.uk/bsn/m621.html

Jiang, S., Cao, Y., Lyenger, S., Kuryloski, P., Jafari, R., Xue, Y., Bajcsy, R., \& Wicker, S. (2008) CareNet: an integrated wireless sensor networking environment for remote healthcare. In: Proc. of nternational conference on body area networks. Tempe, Arizona. https://doi.org/10.4108/ICST.BODYNETS2008.2965

Younis, M., Akkaya, K. et al (2004) On handling QoS traffic in wireless sensor network. In: receedings of the 37th annual Hawaii international conference on system sciences. Hawaii. https://doi.org/10.1109/HICSS.2004.1265688

Patel, M., \& Wang, J. (2010) Applications, challenges, and prospective in emerging body area networking technologies. IEEE Wirel Commun Mag, 17(1), 80-88. https://doi.org/10.1109/MWC.2010.5416354

Zhen, B., Patel, M., Lee, S., Won, E., \& Astrin, A. (2008) TG6 technical requirements document (TRD) IEEE P802.15-08-0644-09-0006. http://mentor.ieee.org/802.15.

Taparugssanagorn, A., Rabbachin, A., Hamalainen, M., Saloranta, J., \& Iinatti, J. (2008) A review of hannel 
modeling for wireless body area network in wireless medical communications. In: The 1th international symposium on wireless personal multimedia communications. Saariselka, Finland.

Yazdandoost, K., \& Sayrafian-Pour, K. (2009) Channel model for body area network (BSN). Doc. \# EEE P802.15-08-0780-06-0006. Available online at http://mentor.ieee.org/802015/

\section{Copyrights}

Copyright for this article is retained by the author(s), with first publication rights granted to the journal.

This is an open-access article distributed under the terms and conditions of the Creative Commons Attribution license (http://creativecommons.org/licenses/by/4.0/). 